\title{
A Prognostic Predictive System Based on Deep Learning for Locoregionally Advanced Nasopharyngeal Carcinoma
}

\author{
Mengyun Qiang, PhD, ${ }^{1}$ Chaofeng Li, PhD, ${ }^{2, \dagger}$ Yuyao Sun (D), MD, ${ }^{3, \dagger}$ Ying Sun $\mathbb{D}, \mathrm{PhD},{ }^{6}$ Liangru Ke, PhD, \\ Chuanmiao Xie, MD, ${ }^{7}$ Tao Zhang (D), MD, ${ }^{8}$ Yujian Zou, BS, ${ }^{9}$ Wenze Qiu, PhD, ${ }^{10}$ Mingyong Gao, MD, ${ }^{11}$ \\ Yingxue Li, MD, ${ }^{3}$ Xiang Li, PhD, ${ }^{3}$ Zejiang Zhan (D) MD, ${ }^{10}$ Kuiyuan Liu, PhD, ${ }^{1}$ Xi Chen $\left(\mathbb{D}, \mathrm{MD},{ }^{1}\right.$ \\ Chixiong Liang, MD, ${ }^{1}$ Qiuyan Chen, $\mathrm{PhD},{ }^{1}$ Haiqiang Mai, $\mathrm{PhD},{ }^{1}$ Guotong Xie, $\mathrm{PhD},{ }^{3,4,5, *}$ Xiang Guo, $\mathrm{PhD},{ }^{1, *}$ \\ Xing Lv (D), $\mathrm{PhD}^{1, *}$
}

\begin{abstract}
${ }^{1}$ Department of Nasopharyngeal Carcinoma, Sun Yat-sen University Cancer Center, Guangzhou, Guangdong, China; ${ }^{2}$ Department of Artificial Intelligence Laboratory, Sun Yat-sen University Cancer Center, Guangzhou, Guangdong, China; ${ }^{3}$ Ping An Healthcare Technology, Beijing, China; ${ }^{4}$ Ping An Health Cloud Company Limited, Beijing, China; ${ }^{5}$ Ping An International Smart City Technology Co., Ltd., Beijing, China; ${ }^{6}$ Department of Radiotherapy, Sun Yat-sen University Cancer Center, Guangzhou, Guangdong, China; ${ }^{7}$ Department of Radiology, Sun Yat-sen University Cancer Center, Guangzhou, Guangdong, China; ${ }^{8}$ Department of Information, The Affiliated Nanfang Hospital of Southern Medical University, Guangzhou, Guangdong, China; ${ }^{9}$ Department of Radiology, The People's Hospital of Dongguan, Dongguan, Guangdong, China; ${ }^{10}$ Department of Radiotherapy, The Affiliated Cancer Hospital of Guangzhou Medical University, Guangzhou, Guangdong, China and ${ }^{11}$ Department of Radiology, The First People's Hospital of Foshan, Foshan, Guangdong, China

${ }^{*}$ Correspondence to: Xing Lv, PhD, Department of Nasopharyngeal Carcinoma, Sun Yat-sen University Cancer Center, No. 651 Dongfeng Road East, 510060 Guangzhou, China (e-mail: lvxing@sysucc.org.cn) and Xiang Guo, PhD, Department of Nasopharyngeal Carcinoma, Sun Yat-sen University Cancer Center, No. 651 Dongfeng Road East, 510060 Guangzhou, China (e-mail: guoxiang@sysucc.org.cn) and Guotong Xie, PhD, Ping An Healthcare Technology, No. 23 Financial Street, 100032, Beijing, China (e-mail: xieguotong@pingan.com.cn).
\end{abstract}

${ }^{\dagger}$ Mengyun Qiang, Chaofeng Li and Yuyao Sun contributed equally to this work.

\begin{abstract}
Background: Images from magnetic resonance imaging (MRI) are crucial unstructured data for prognostic evaluation in nasopharyngeal carcinoma (NPC). We developed and validated a prognostic system based on the MRI features and clinical data of locoregionally advanced NPC (LA-NPC) patients to distinguish low-risk patients with LA-NPC for whom concurrent chemoradiotherapy (CCRT) is sufficient. Methods: This multicenter, retrospective study included 3444 patients with LA-NPC from January 1, 2010, to January 31, 2017. A 3-dimensional convolutional neural network was used to learn the image features from pretreatment MRI images. An eXtreme Gradient Boosting model was trained with the MRI features and clinical data to assign an overall score to each patient. Comprehensive evaluations were implemented to assess the performance of the predictive system. We applied the overall score to distinguish high-risk patients from low-risk patients. The clinical benefit of induction chemotherapy (IC) was analyzed in each risk group by survival curves. Results: We constructed a prognostic system displaying a concordance index of 0.776 (95\% confidence interval $[\mathrm{CI}]=0.746$ to 0.806$)$ for the internal validation cohort and 0.757 ( $95 \% \mathrm{CI}=0.695$ to 0.819 ), 0.719 ( $95 \% \mathrm{CI}=0.650$ to 0.789 ), and 0.746 ( $95 \% \mathrm{CI}=0.699$ to 0.793 ) for the 3 external validation cohorts, which presented a statistically significant improvement compared with the conventional TNM staging system. In the high-risk group, patients who received induction chemotherapy plus CCRT had better outcomes than patients who received CCRT alone, whereas there was no statistically significant difference in the low-risk group. Conclusions: The proposed framework can capture more complex and heterogeneous information to predict the prognosis of patients with LA-NPC and potentially contribute to clinical decision making.
\end{abstract}

Nasopharyngeal carcinoma (NPC) is an invasive epithelial malignancy. In 2018, there were an estimated 130000 incident cases worldwide, with high prevalence rates in South China, Southeastern Asia, and North Africa (1). More than $75 \%$ of patients are diagnosed with locoregionally advanced NPC (LANPC) (TNM stage III or IVA) at the first visit (2). Approximately $30 \%$ of these patients eventually suffer from treatment failure after being administered with radical chemoradiotherapy (3). 
Stage III and IVA patients are recommended induction chemotherapy (IC) plus concurrent chemoradiotherapy (CCRT) as $2 \mathrm{~A}$ level evidence, based on the National Comprehensive Cancer Network guidelines (4). However, uncertainty remains regarding whether all of these stage III and IVA patients need aggressive treatment strategies. Prior studies attempted to investigate new predictive methods to distinguish low-risk patients with LANPC, for whom CCRT was sufficient (5-8).

The TNM staging system is the key criterion for prognostic prediction and risk stratification for treatment decisions and is mainly dependent on anatomical information such as the tumor involvement of adjacent tissue detected by magnetic resonance imaging (MRI). However, different prognoses are observed in patients within the same TNM stage after receiving equivalent standard treatments (9). A potential reason is that the TNM staging system might have insufficient predictive power for prognosis. To improve the prognostic performance of the TNM staging system, some researchers applied apparent diffusion coefficient (ADC) parameters or MRI features in comprehensive predictive models (7,10-12), and others applied clinical indicators, such as Epstein-Barr virus (EBV) DNA, age, and body mass index (BMI), all of which are closely related to the prognosis of NPC patients $(13,14)$.

Although several prognostic models have improved the accuracy of prediction, by applying MRI data in the form of $A D C$ parameters or MRI features (7,10-12), they all require MRI images to be manually segmented, which is complex and inconvenient for practical applications. In addition, ADC parameters require extra magnetic resonance (MR) scanning sequence-diffusionweighted images. MRI features are often extracted by unsupervised learning procedures, resulting in many features being less correlated with the endpoint. Regarding the application of clinical indicators, the existing predictive models are often based on linear approaches, which insufficiently explain the nonlinear relationship between factors and outcomes and thus have less accurate performance $(13,14)$. Currently, deep learning methods, especially convolutional neural networks (CNNs), are commonly applied to analyze nonstructural image data and have shown powerful image-feature capturing capacities $(15,16)$. Machine learning techniques such as eXtreme Gradient Boosting (XGBoost) (17) are good at modeling nonlinear relationships in structural data and have achieved state-of-the-art results in different fields $(18,19)$.

In this multicenter study, we constructed a framework to predict disease-free survival (DFS) in LA-NPC patients with the goal of overcoming the previously mentioned limitations in existing prognostic models. We aimed to 1) extract MRI features by the 3-dimensional (3D) CNN model; 2) integrate image features and structural clinical factors by the XGBoost model to assign an overall score to each patient; and 3) classify LA-NPC patients into high-risk and low-risk groups based on the cutoff value of the overall score and analyze the survival benefits of IC in different risk groups.

\section{Methods}

\section{Patients and Datasets}

The clinical data and MRI images of patients were collected from 4 hospitals. There were 1838, 787, 257, 248, and 314 LANPC patients in the training cohort, the internal validation cohort, and external validation cohorts 1,2 , and 3, respectively. The training and internal validation cohorts were obtained by randomly splitting the patients from 1 hospital. Details on the dataset, inclusion criteria, therapeutic regimens, follow-up dates, and random splitting method are provided in the Supplementary Methods (available online).

Three sequences (axial T1-weighted [T1w], T2-weighted [T2w], and $\mathrm{T} 1 \mathrm{w}$ contrast [T1c]) of pretreatment head and neck MRI were collected. The MRI scanning protocol is detailed in the Supplementary Methods (available online). The acquisition parameters are shown in Supplementary Table 1 (available online).

Eight clinical features were collected: EBV DNA, T stage, $\mathrm{N}$ stage, sex, age, BMI, albumin (ALB), and serum lactate dehydrogenase (LDH). Of note, EBV DNA was unavailable in external validation cohort 2 . The patients were restaged by 2 radiologists according to the 8th Edition of the American Joint Committee on Cancer-Union for International Cancer Control staging system (20). Informed consent was obtained, and chart reviews were performed after approval by the China Ethics Committee of Registering Clinical Trials (No. ChiECRCT20190036).

\section{Prognostic Predictive System}

The workflow of the prognostic prediction system is illustrated in Figure 1 and consists of 2 main steps.

First, the 3D-CNN was employed to learn MRI features. Pretreatment MRI images without any local annotation were preprocessed to unify the pixel size and filter the images. The 3D-CNN was trained with the normalized MRI images and corresponding DFS information (DFS month and DFS status). We named the learned MRI features by 3D-CNN the "MRI score." The details of how we preprocessed the images and constructed the 3D-CNN model are described in the Supplementary Methods (available online). The pipeline of the 3D-CNN is presented in Supplementary Figure 1 (available online).

Second, the XGBoost model was trained with 9 features (MRI score, EBV DNA, T stage, N stage, sex, age, BMI, ALB, and $\mathrm{LDH})$, which learned the nonlinear relationship between DFS and the combined features. XGBoost assigned an overall score to each patient. Of note, because XGBoost is able to deal with the missing values automatically, our model can still be applied on the external validation cohort 2 without EBV DNA. Further details of the XGBoost model and how they handle the missing values are described in the Supplementary Methods (available online). The hyperparameters in the final proposed XGBoost model are presented in Supplementary Table 2 (available online).

\section{Statistical Analysis}

We compared the predictive performance of the TNM stage, MRI score, and overall score by the concordance index (C-index) (21) and time-dependent area under the curve (AUC) (22) values. To assess whether the improvements in the $\mathrm{C}$-indexes and the time-dependent AUC values between the compared models (MRI score vs TNM stage, overall score vs TNM stage) were statistically significant, we calculated the $P$ values using the "survcomp" (23) and "timeROC" (24) packages in R. A detailed description of how we calculated the C-index can be found in the Supplementary Methods (available online).

We calculated the optimal cutoff value for the overall score using X-tile software (v3.6.1; Yale University, CT) in the training cohort. Then, the patients were stratified into high- and low- 


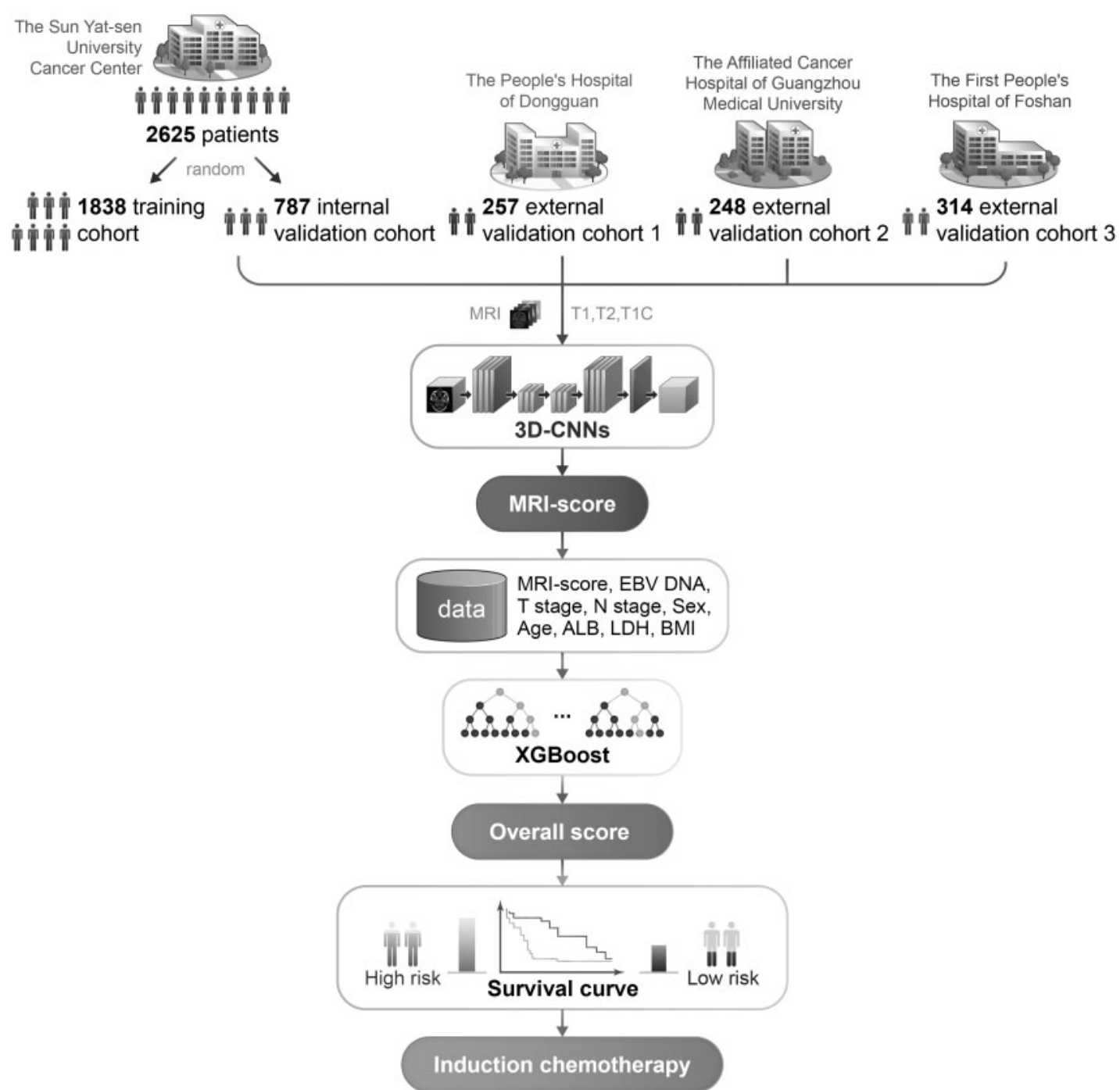

Figure 1. Research layout. Patients were enrolled from 4 hospitals (the Sun Yat-sen University Cancer Center, the People's Hospital of Dongguan, the Affiliated Cancer Hospital of Guangzhou Medical University, and The First People's Hospital of Foshan). First, we trained the 3D-CNN model on 3 MRI sequences (axial T1, T2, and T1c) to predict disease-free survival in the training cohort. An internal validation cohort and 3 external validation cohorts validated the robustness of the 3D-CNN model. The MRI score extracted from the 3D-CNN model was assigned to each patient. Then, we built an XGBoost model with 9 features (MRI score, EBV DNA, T stage, N stage, sex, age, ALB, LDH, and BMI). The overall score extracted from the XGBoost model was assigned to each patient. The cutoff value of the overall score was used to separate patients into high- and low-risk groups. Finally, we compared the survival rates of patients who received induction chemotherapy with those of patients who did not receive induction chemotherapy in different risk level groups. 3D-CNN = 3-dimensional convolutional neural network; $\mathrm{ALB}=$ albumin; $\mathrm{BMI}=$ body mass index; $\mathrm{DNA}=$ deoxyribonucleic acid; $\mathrm{EBV}=$ Epstein-Barr virus; $\mathrm{LDH}=$ serum lactate dehydrogenase; $\mathrm{N}=$ node; $\mathrm{MRI}=$ magnetic resonance imaging; $\mathrm{T} 1=\mathrm{T} 1 \mathrm{weighted} ; \mathrm{T} 2=\mathrm{T} 2$ weighted; $\mathrm{T}=$ tumor; $\mathrm{XGBoost}=$ eXtreme Gradient Boosting.

risk groups. We plotted Kaplan-Meier curves and calculated the hazard ratios (HRs) to compare the survival conditions between the high- and low-risk groups. The same analysis was also implemented for TNM stage (stage III is low-risk, and stage IVA is high risk) for comparison.

To assess the effect of the proposed risk stratification strategy (based on the overall score) on the treatment decision, we further compared the Kaplan-Meier curves of the patients receiving IC plus CCRT with those of patients receiving CCRT in the low- and high-risk groups separately.

The log-rank test was applied for comparisons between different survival curves. The hazard ratio estimated by univariate Cox regression was also used to evaluate the difference in survival curves. The statistical significance test for comparing 2 hazard ratios was implemented using generic inverse variance meta-analysis in the R package "meta."

The primary endpoint, DFS, was defined as the time from antitumor treatment to disease progression or death from any cause. The secondary endpoints were overall survival (OS; the time from antitumor treatment to death from any cause) and distant metastasis-free survival (DMFS; the time from antitumor treatment to distant metastasis).

Differences in the clinical features of the 4 validation cohorts were analyzed using the nonparametric Kruskal-Wallis test and $\chi^{2}$ test. All statistical tests were implemented using R (v3.6.0) and SPSS (v25.0). All tests were 2-sided for statistical significance testing, and a Pvalue of less than .05 was defined as the statistical significance threshold. 


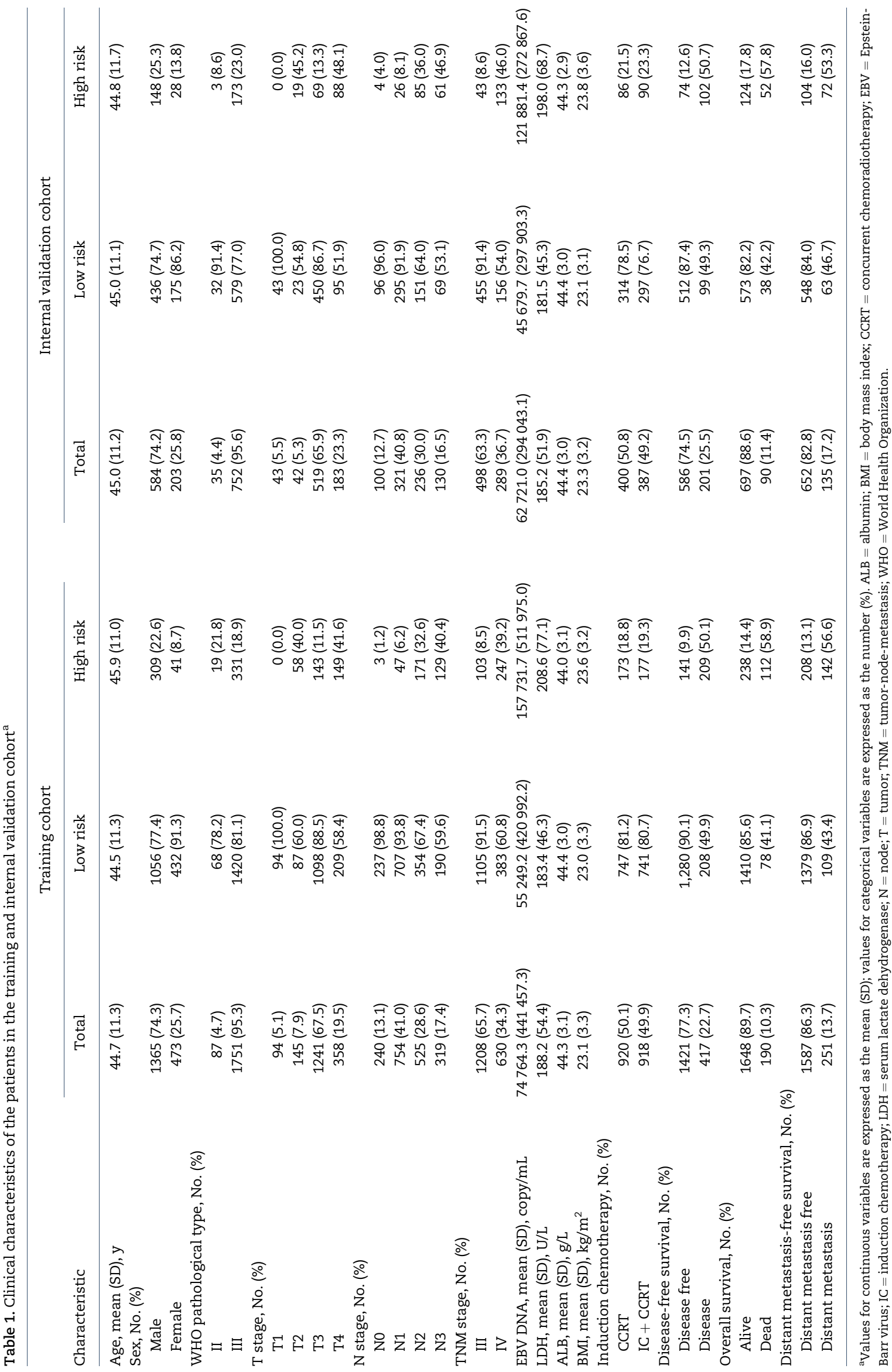




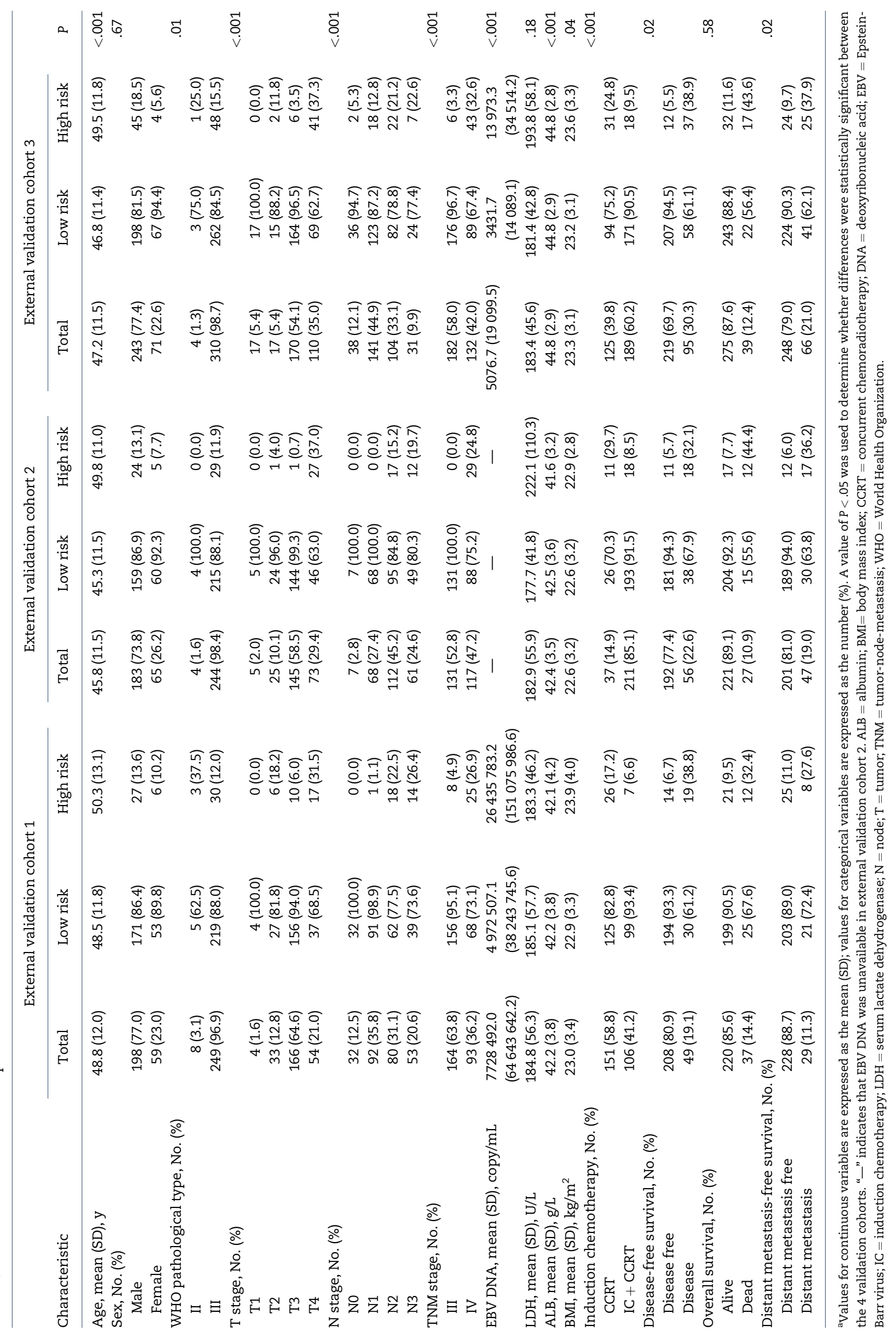


Table 3. Concordance indexes of TNM stage, MRI score, and overall score for the training cohort and validation cohorts

\begin{tabular}{|c|c|c|c|c|c|c|c|c|}
\hline \multirow[b]{2}{*}{ Factor } & \multicolumn{2}{|l|}{ DFS } & \multicolumn{3}{|c|}{ Os } & \multicolumn{3}{|c|}{ DMFS } \\
\hline & C-index $(95 \%$ CI) & $P^{\mathrm{a}}$ & C-index & $95 \%$ CI & $P^{\mathrm{a}}$ & C-index & $95 \%$ CI & $P^{a}$ \\
\hline \multicolumn{9}{|l|}{ Training cohort } \\
\hline TNM stage & 0.638 (0.614 to 0.662$)$ & Referent & $0.622(0.5$ & to 0.657$)$ & Referent & $0.648(0.6$ & to 0.677 ) & Referent \\
\hline MRI score & 0.700 (0.675 to 0.726$)$ & $<.001$ & $0.738(0.7$ & to 0.771 ) & $<.001$ & $0.712(0.6$ & to 0.743 ) & .003 \\
\hline Overall score & 0.787 (0.767 to 0.806$)$ & $<.001$ & $0.817(0.7$ & to 0.842$)$ & $<.001$ & $0.806(0.7$ & to 0.803$)$ & $<.001$ \\
\hline \multicolumn{9}{|c|}{ Internal validation cohort } \\
\hline TNM stage & 0.633 (0.600 to 0.666$)$ & Referent & $0.627(0.5$ & to 0.678$)$ & Referent & $0.650(0.6$ & to 0.691 ) & Referent \\
\hline MRI score & 0.694 (0.659 to 0.729$)$ & .01 & $0.717(0.6$ & to 0.764 ) & .009 & $0.692(0.6$ & to 0.733$)$ & .10 \\
\hline Overall score & 0.776 (0.746 to 0.806$)$ & $<.001$ & $0.800(0.7$ & to 0.840 ) & $<.001$ & $0.782(0.7$ & to 0.818 ) & $<.001$ \\
\hline \multicolumn{9}{|c|}{ External validation cohort 1} \\
\hline TNM stage & 0.625 (0.566 to 0.694$)$ & Referent & $0.632(0.5$ & to 0.710 ) & Referent & $0.613(0.5$ & to 0.703 ) & Referent \\
\hline MRI score & 0.690 (0.620 to 0.761$)$ & .10 & $0.671(0.5$ & to 0.775 ) & .26 & $0.640(0.5$ & to 0.732 ) & .35 \\
\hline Overall score & 0.757 (0.695 to 0.819$)$ & $<.001$ & $0.745(0.6$ & to 0.816 ) & .001 & $0.696(0.6$ & to 0.785 ) & .03 \\
\hline \multicolumn{9}{|c|}{ External validation cohort 2} \\
\hline TNM stage & 0.627 (0.566 to 0.688$)$ & Referent & $0.690(0.6$ & to 0.763 ) & Referent & $0.645(0.5$ & to 0.710$)$ & Referent \\
\hline MRI score & 0.685 (0.611 to 0.760$)$ & .13 & $0.755(0.6$ & to 0.859$)$ & .17 & $0.683(0.6$ & to 0.763 ) & .25 \\
\hline Overall score & 0.719 (0.650 to 0.789$)$ & .002 & $0.826(0.7$ & to 0.895$)$ & $<.001$ & $0.718(0.6$ & to 0.795$)$ & .02 \\
\hline \multicolumn{9}{|c|}{ External validation cohort 3} \\
\hline TNM stage & 0.628 (0.579 to 0.677$)$ & Referent & $0.660(0.5$ & to 0.734 ) & Referent & $0.640(0.5$ & to 0.699 ) & Referent \\
\hline MRI score & 0.695 (0.644 to 0.746$)$ & .05 & $0.760(0.6$ & to 0.834 ) & .05 & $0.711(0.6$ & to 0.774 ) & .07 \\
\hline Overall score & 0.746 (0.699 to 0.793$)$ & $<.001$ & $0.780(0.7$ & to 0.849 ) & $<.001$ & $0.768(0.7$ & to 0.817$)$ & $<.001$ \\
\hline
\end{tabular}

${ }^{\mathrm{a}} \mathrm{A}$ value of $\mathrm{P}<.05$ was used to determine whether differences were statistically significant between the predictive models and TNM stage. C-index $=$ concordance index; $\mathrm{CI}=$ confidence interval; DFS = disease-free survival; DMFS = distant metastasis-free survival; MRI = magnetic resonance imaging; OS = overall survival; TNM stage $=$ tumor-node-metastasis stage.

\section{Results}

\section{Patient Characteristics and Follow-up}

We identified 3444 eligible patients from 4 hospitals. The clinical characteristics of the patients are presented in Tables 1 and 2 . Among the 4 validation cohorts, no statistically significant difference was observed for sex and LDH, whereas age, T stage, $\mathrm{N}$ stage, TNM stage, EBV DNA, BMI, and ALB were statistically significantly different. The numbers of patients with DFS, OS, and DMFS outcomes in the low- and high-risk groups are presented in Tables 1 and 2.

\section{Comparison of the Predictive Accuracy of TNM Stage, MRI Score, and Overall Score}

The C-indexes of TNM stage in predicting DFS were $0.638(95 \%$ confidence interval $[\mathrm{CI}]=0.614$ to 0.662$), 0.633(95 \% \mathrm{CI}=0.600$ to $0.666), 0.625$ (95\% CI $=0.566$ to 0.694$), 0.627$ (95\% CI $=0.566$ to 0.688 ), and 0.628 (95\% CI $=0.579$ to 0.677 ) for the training cohort, the internal validation cohort, and external validation cohorts 1,2 , and 3 , respectively. These values were all lower than those of the MRI score (Table 3). The C-index of the MRI score for predicting DFS in the combined external validation cohorts 1,2 , and 3 was statistically significantly higher than that of TNM stage $(0.691,95 \% \mathrm{CI}=0.656$ to 0.726 vs $0.629,95 \% \mathrm{CI}=0.596$ to 0.662; $P=.01)$. The $\mathrm{C}$-indexes for the overall score in predicting DFS were statistically significantly higher than those of TNM stage: 0.787 (95\% CI $=0.767$ to $0.806 ; P<.001), 0.776(95 \% \mathrm{CI}=$ 0.746 to $0.806 ; P<.001), 0.757(95 \% \mathrm{CI}=0.695$ to $0.819 ; \mathrm{P}<.001)$, 0.719 ( $95 \% \mathrm{CI}=0.650$ to $0.789 ; \mathrm{P}=.002)$, and 0.746 ( $95 \% \mathrm{CI}=0.699$ to $0.793 ; \mathrm{P}<.001$ ) for each corresponding cohort. The $\mathrm{C}$-indexes of the MRI score, the overall score, and TNM stage in predicting OS and DMFS are presented in Table 3.
We calculated the 5-year time-dependent AUC to compare the predictive accuracy of TNM stage, MRI score, and overall score. The AUCs of TNM stage were $0.674(95 \% \mathrm{CI}=0.631$ to $0.717), 0.612(95 \% \mathrm{CI}=0.521$ to 0.704$), 0.613(95 \% \mathrm{CI}=0.517$ to 0.708 ), and 0.675 ( $95 \% \mathrm{CI}=0.609$ to 0.740 ) for the internal validation cohort and external validation cohorts 1,2 , and 3, respectively. They were lower than those of the MRI score, and statistically significantly lower than those of overall score for each corresponding validation cohort (Table 4). Table 4 and Supplementary Table 3 (available online) present the timedependent AUC values of TNM stage, the MRI score, and the overall score in predicting 5-year and 3-year DFS, OS, and DMFS. Supplementary Figures 2 and 3 (available online) present the time-dependent ROC curves for TNM stage, the MRI score, and the overall score in predicting 3-year and 5-year DFS, OS, and DMFS.

Comparison of the Discrimination Ability of TNM Stage and Overall Score in the High- and Low-Risk Groups

We categorized the patients into high- and low-risk groups according to the optimal cutoff value of the overall score (0.95) determined by $\mathrm{X}$-tile software in the training cohort (Supplementary Figure 4, available online). Patients with an overall score higher than the cutoff value were divided into the high-risk group, and the other patients were divided into the low-risk group. There were 1488, 611, 224, 219, and 265 low-risk patients in the training cohort, the internal validation cohort, and external validation cohorts 1,2 , and 3, respectively. In addition, 350, 176, 33, 29, and 49 high-risk patients were included in the training cohort, the internal validation cohort, and external validation cohorts 1, 2, and 3, respectively. High-risk patients had poorer DFS than low-risk patients $(\mathrm{HR}=6.05,95 \% \mathrm{CI}=4.99$ to $7.34 ; \mathrm{P}<.001 ; \mathrm{HR}=4.99,95 \% \mathrm{CI}=3.78$ to $6.59 ; \mathrm{P}<.001$; 
Table 4. Five-year time-dependent AUCs of TNM stage, MRI score, and overall score for the training cohort and validation cohorts

\begin{tabular}{|c|c|c|c|c|c|c|}
\hline \multirow[b]{2}{*}{ Factor } & \multicolumn{2}{|l|}{ DFS } & \multicolumn{2}{|l|}{ os } & \multicolumn{2}{|l|}{ DMFS } \\
\hline & AUC $(95 \%$ CI $)$ & $P^{\mathrm{a}}$ & AUC $(95 \%$ CI) & $P^{\mathrm{a}}$ & AUC $(95 \%$ CI $)$ & $P^{a}$ \\
\hline \multicolumn{7}{|l|}{ Training cohort } \\
\hline TNM stage & 0.668 (0.640 to 0.697$)$ & Referent & 0.642 (0.602 to 0.682$)$ & Referent & 0.674 (0.639 to 0.709$)$ & Referent \\
\hline MRI score & 0.692 (0.660 to 0.723$)$ & .29 & 0.721 (0.683 to 0.759$)$ & .006 & 0.705 (0.669 to 0.740$)$ & .23 \\
\hline Overall score & 0.809 (0.783 to 0.834$)$ & $<.001$ & 0.816 (0.784 to 0.847$)$ & $<.001$ & 0.822 (0.793 to 0.852$)$ & $<.001$ \\
\hline \multicolumn{7}{|c|}{ Internal validation cohort } \\
\hline TNM stage & 0.674 (0.631 to 0.717$)$ & Referent & 0.621 (0.563 to 0.679$)$ & Referent & 0.684 (0.636 to 0.732$)$ & Referent \\
\hline MRI score & 0.690 (0.643 to 0.737$)$ & .61 & 0.689 (0.633 to 0.746$)$ & .07 & 0.680 (0.628 to 0.732$)$ & .92 \\
\hline Overall score & 0.806 (0.768 to 0.845$)$ & $<.001$ & $0.787(0.735$ to 0.838$)$ & $<.001$ & 0.798 (0.754 to 0.842$)$ & $<.001$ \\
\hline \multicolumn{7}{|c|}{ External validation cohort 1} \\
\hline TNM stage & 0.612 (0.521 to 0.704$)$ & Referent & $0.618(0.517$ to 0.718$)$ & Referent & 0.607 (0.500 to 0.714$)$ & Referent \\
\hline MRI score & 0.691 (0.592 to 0.790$)$ & .25 & 0.655 (0.546 to 0.765$)$ & .62 & 0.641 (0.526 to 0.756$)$ & .68 \\
\hline Overall score & 0.759 (0.664 to 0.854$)$ & .001 & 0.728 (0.618 to 0.838$)$ & .03 & 0.705 (0.598 to 0.812$)$ & .06 \\
\hline \multicolumn{7}{|c|}{ External validation cohort 2} \\
\hline TNM stage & 0.613 (0.517 to 0.708$)$ & Referent & 0.658 (0.552 to 0.764$)$ & Referent & 0.625 (0.526 to 0.724$)$ & Referent \\
\hline MRI score & 0.749 (0.655 to 0.843$)$ & .05 & 0.813 (0.705 to 0.921$)$ & .07 & $0.740(0.640$ to 0.840$)$ & .10 \\
\hline Overall score & 0.756 (0.663 to 0.849$)$ & .003 & 0.837 (0.752 to 0.922$)$ & .002 & $0.734(0.628$ to 0.839$)$ & .03 \\
\hline \multicolumn{7}{|c|}{ External validation cohort 3} \\
\hline TNM stage & 0.675 (0.609 to 0.740$)$ & Referent & 0.672 (0.584 to 0.761$)$ & Referent & 0.688 (0.616 to 0.760$)$ & Referent \\
\hline MRI score & 0.833 (0.777 to 0.889$)$ & $<.001$ & $0.874(0.821$ to 0.928$)$ & $<.001$ & 0.834 (0.771 to 0.896$)$ & .004 \\
\hline Overall score & 0.857 (0.804 to 0.910$)$ & $<.001$ & 0.853 (0.789 to 0.917$)$ & $<.001$ & 0.868 (0.818 to 0.920$)$ & $<.001$ \\
\hline
\end{tabular}

${ }^{\mathrm{a}} \mathrm{A}$ value of $\mathrm{P}<.05$ was used to determine whether differences were statistically significant between the predictive models and TNM stage. AUC $=$ area under the curve; $\mathrm{CI}=$ confidence interval; DFS = disease-free survival; DMFS = distant metastasis-free survival; MRI = magnetic resonance imaging; OS = overall survival; TNM stage = tumor-node-metastasis stage.

$\mathrm{HR}=5.78,95 \% \mathrm{CI}=3.24$ to $10.29 ; \mathrm{P}<.001 ; \mathrm{HR}=5.21,95 \% \mathrm{CI}=$ 2.96 to $9.16 ; P<.001 ;$ and $\mathrm{HR}=6.15,95 \% \mathrm{CI}=4.01$ to $9.42 ; \mathrm{P}<$ .001 , in the training cohort, the internal validation cohort, and external validation cohorts 1, 2, and 3, respectively) (Supplementary Table 4, available online). We also evaluated the performance of TNM stage in distinguishing the high- and low-risk groups. Stage IVA patients had poorer DFS than stage III patients $(\mathrm{HR}=3.18,95 \% \mathrm{CI}=2.62$ to $3.86 ; \mathrm{P}<.001 ; \mathrm{HR}=3.09$, $95 \% \mathrm{CI}=2.33$ to $4.09 ; \mathrm{P}<.001 ; \mathrm{HR}=2.65,95 \% \mathrm{CI}=1.51$ to $4.68 ; \mathrm{P}$ $<.001 ; \mathrm{HR}=2.91,95 \% \mathrm{CI}=1.65$ to $5.15 ; \mathrm{P}<.001$; and $\mathrm{HR}=2.73$, $95 \% \mathrm{CI}=1.80$ to $4.14 ; \mathrm{P}<.001$, in the training cohort, the internal validation cohort, and external validation cohorts 1,2 , and 3 , respectively) (Supplementary Table 4, available online). The XGBoost model had a statistically significantly higher hazard ratio value than the TNM stage model, with $P$ value less than .001 in the training cohort, $P$ value equal to .02 in the internal validation cohort, and $P$ value less than .001 in the combined external validation cohorts 1, 2, and 3. In the Kaplan-Meier curves, we observed a more obvious distinction between high- and lowrisk group patients based on the overall score than based on TNM stage (Figure 2). Supplementary Figure 5 (available online) presents the Kaplan-Meier curves for the OS and DMFS outcomes of the high- and low-risk groups based on the overall score. These findings suggest that compared with TNM stage, the overall score more effectively distinguishes LA-NPC patients with good prognosis from those with poor prognosis.

\section{Different Survival Benefits of IC in the High- and Low- Risk Groups Based on Overall Score}

High-risk patients were distinguished from low-risk patients based on the optimal cutoff value of the overall score. We further investigated whether IC was necessary in different risk groups by comparing the survival curves between patients treated with IC plus CCRT and those treated with CCRT alone. In the high-risk group, patients who received IC plus CCRT had better DFS than patients who received CCRT alone (HR $=0.37$, $95 \% \mathrm{CI}=0.28$ to $0.49 ; \mathrm{P}<.001 ; \mathrm{HR}=0.46,95 \% \mathrm{CI}=0.31$ to 0.69 ; $P<.001$; and $\mathrm{HR}=0.32,95 \% \mathrm{CI}=0.19$ to $0.54 ; \mathrm{P}<.001$, in the training cohort, internal validation cohort, and combined external validation cohorts 1, 2, and 3, respectively). However, in the low-risk group, patients who were treated with IC plus CCRT did not have better DFS than patients who were treated with CCRT alone $(\mathrm{HR}=0.86,95 \% \mathrm{CI}=0.65$ to $1.13 ; \mathrm{P}=.28 ; \mathrm{HR}=0.68,95 \% \mathrm{CI}$ $=0.45$ to $1.01 ; \mathrm{P}=.06$; and $\mathrm{HR}=1.02,95 \% \mathrm{CI}=0.70$ to $1.48 ; \mathrm{P}=$ .92 , in the training cohort, internal validation cohort, and combined external validation cohorts 1,2 and 3, respectively; Figure 3). These findings were also observed for OS and DMFS (Supplementary Table 5 and Supplementary Figures 6-8, available online). These results indicated that the overall score is a reliable prognostic factor that can be used to select patients at high risk who might benefit from IC.

\section{Discussion}

In this multicenter study, we developed a prognostic predictive system to accurately predict the DFS of NPC by integrating MR image features of the primary lesion and important clinical characteristics. The predictive performance of the overall score and the MRI score were better than that of the 8th TNM staging system. These promising results mainly stem from the following reasons. MRI is crucial for evaluating primary tumor extension and detecting retropharyngeal and cervical lymph node metastasis compared with other imaging modalities (25). The $3 \mathrm{D}-\mathrm{CNN}$ showed the capacity to extract complex and informative features from MRI, such as texture and grayscale. Some MRI features are closely tied to patient outcomes, as observed in a study by LL Tang, et al. (7), who proposed a prognostic model 
A Training cohort: 1838 patients

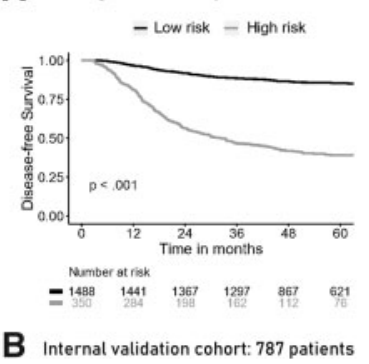

Internal validation cohort: 787 patie
- Low risk - High risk
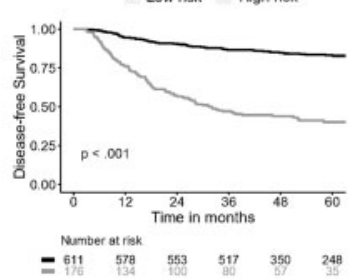

C External validation cohort 1: 257 patients
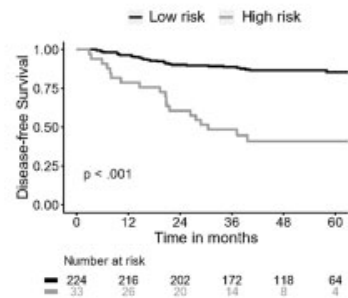

D External validation cohort 2:248 patients
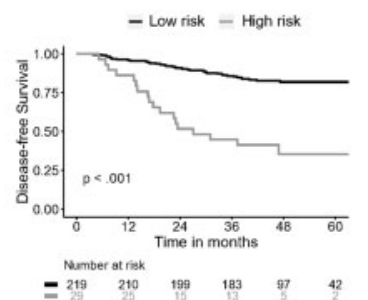

E External validation cohort 3: 314 patients

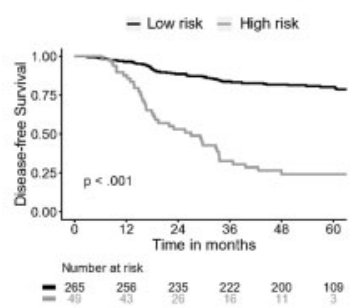

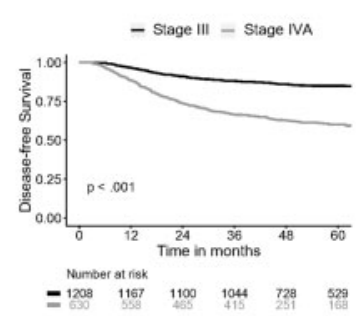
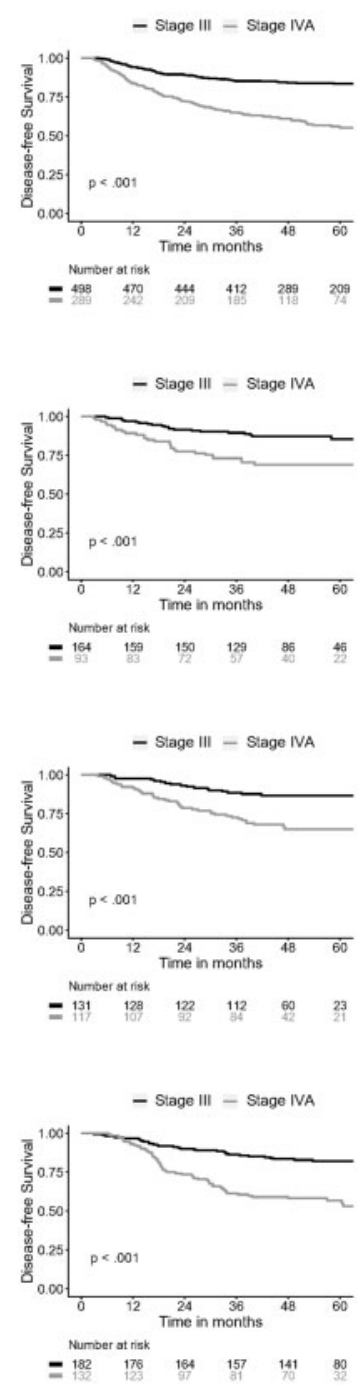

Figure 2. Kaplan-Meier curves of the High- and Low-risk groups based on the eXtreme Gradient Boosting model and tumor-node-metastasis staging system. Kaplan-Meier plots are shown for the disease-free survival outcomes of the High-risk and Low-risk groups in (A) the training cohort, (B) the internal validation cohort, (C) the external validation cohort 1, (D) the external validation cohort 2, and (E) the external validation cohort 3. The optimal cutoff value of the overall score was 0.95 , as selected by X-tile. Patients with an overall score higher than the cutoff value were divided into the High-risk group, whereas the other patients were divided into the Low-risk group. For the tumor-node-metastasis staging system, patients in Stage III were considered low-risk, whereas those in Stage IVA were considered high-risk.

based on MRI radiomics features in LA-NPC. Similar conclusions have been indicated in other studies on nasopharyngeal carcinoma $(26,27)$. In contrast, conventional practice, such as the
TNM staging system, merely focuses on specified anatomical information and necrosis position. Moreover, compared with other studies, our research only needed conventional MR scanning sequences. Additionally, we extracted MR image features effectively without additional manual segmentation of the tumor.

The results showed that the overall score of our proposed framework made improvements compared to common practice $(6,13,28)$. This was possibly ascribed to the following reasons First, MRI features were used to the greatest extent in this study. Peng et al. (6) and LL Tang et al. (7) both proposed a prognostic model mainly based on radiomics features. Peng and colleagues' (6) research was mainly based on positron emission tomography-computed tomography radiomics features. However, MRI is better for evaluating primary tumor extension than positron emission tomography-computed tomography. In LL Tang and colleagues' research, only $3 \mathrm{MR}$ image features were selected out of 819 features by the Cox proportional hazards model. The DeepSurvivalNet framework of the 3D-CNN model in our paper could maximize the utilization of the MR image information. Second, we incorporated MRI and clinical factors to predict patient outcomes. The results indicated that these 2 types of information could complement each other to make better predictions. Third, XGBoost is an efficient method to handle nonlinear relationships and missing values compared with nomogram models, which are based on Cox regression models (18). Our findings indicated that integrating the MRI and clinical features could help reveal the extreme variability in LA-NPC patients. Thus, we believe our framework is a practical tool for predicting DFS in NPC patients.

The overall score effectively divided patients into subgroups of different risk levels and helped distinguish which group of patients was suitable for IC. The role of IC in the treatment of LA-NPC patients is a long-standing controversy. According to the National Comprehensive Cancer Network guidelines, IC plus CCRT is recommended as $2 \mathrm{~A}$ level evidence (4). However, not all LA-NPC patients benefit from IC $(29,30)$. These inconsistent results may be related to the rough division of the locally advanced category. Therefore, it is necessary to divide LA-NPC patients into subgroups based on different outcomes and provide individual treatment. In this study, we classified LA-NPC patients with comprehensive algorithms and observed that patients in the high-risk group benefited from IC. It is beneficial to relieve the financial burden of patients in the low-risk group, shorten the waiting time for radiotherapy to reduce mortality risk (31), and avoid concurrent chemotherapy dose reduction due to adverse reactions from IC.

Several limitations must be noted. First, this was a retrospective study, and the integrity and homogeneity of the data had deficiencies (eg, EBV DNA was unavailable in external validation cohort 2, and the laboratory data lacked standardization). Second, deep learning algorithms have limitations in interpretability. Third, the research was conducted in endemic areas and included only World Health Organization pathological types II and III. Fourth, because of the diversity of IC regimens and chemotherapy doses, potential confounding factors were not considered. Although this study had several limitations, our results provide great insights. We applied the XGBoost model to solve the problem of unavailable data, and we validated the robustness of our model in 4 validation cohorts. We plan to conduct a multicenter clinical trial based on our predictive model. Further scientific studies are needed to verify our findings. We also built an online tool for convenient application by clinicians (http://101.89.95.81:8250/swel.html). 
A High-risk group of training cohort: 350 patients

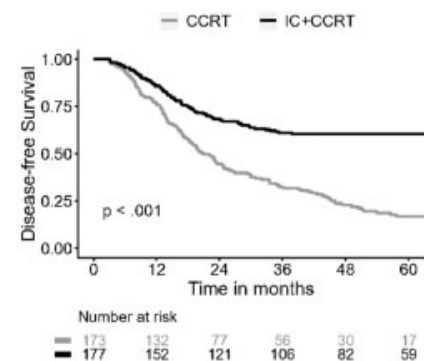

C High-risk group of internal validation cohort: 176 patients

$$
- \text { CCRT } \quad-I C+C C R T
$$
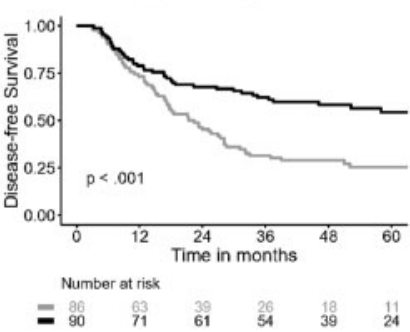

E

High-risk group of combined external validation cohorts 1, 2 and 3: 111 patients
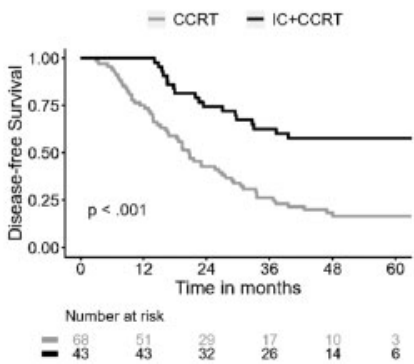

B Low-risk group of training cohort: 1488 patients

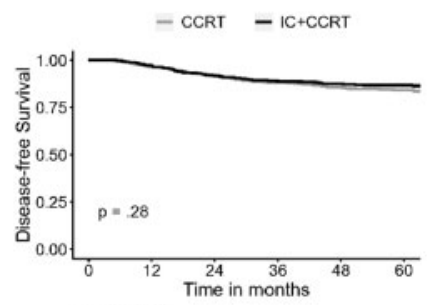

Number at risk

$\begin{array}{llllll}747 & 721 & 666 & 653 & 425 & 314 \\ 742 & 681 & 644 & 442 & 307\end{array}$

B Low-risk group of internal validation cohort 1: 611 patients

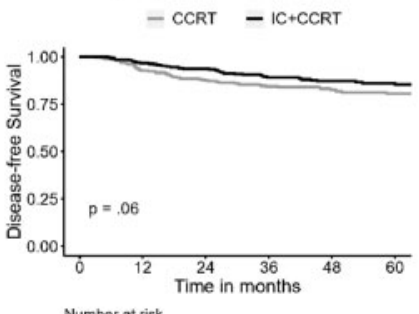

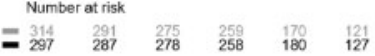

F Low-risk group of combined external validation cohorts

1, 2 and $3: 708$ patients

- CCRT - IC+CCRT

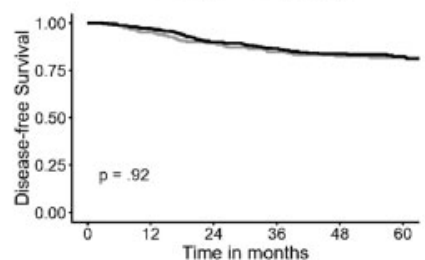

Number at risk

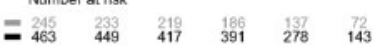

Figure 3. Kaplan-Meier plots of the disease-free survival outcomes of patients receiving induction chemotherapy followed by concurrent chemoradiotherapy and concurrent chemoradiotherapy alone in different risk groups in the 4 cohorts. A) High-risk group of training cohort, (B) low-risk group of the training cohort, (C) high-risk group of the internal validation cohort, (D) low-risk group of the internal validation cohort, (E) high-risk group of combined external validation cohorts, and (F) low-risk group of combined external validation cohorts are shown. The cutoff value of the overall score was 0.95 , as calculated by X-tile. Patients with an overall score higher than the cutoff value were divided into the high-risk group, whereas the other patients were divided into the low-risk group. CCRT = concurrent chemoradiotherapy; IC = induction chemotherapy.

In conclusion, we proposed a prognostic predictive system that integrated the 3D-CNN and XGBoost algorithms. The $3 \mathrm{D}$ CNN effectively revealed the extreme variability in tumor features on MRI. Furthermore, the final overall score, which was outputted from the XGBoost model, shows promise for improving the prediction of survival and for subsequently assisting clinicians in making treatment decisions for LA-NPC patients.

\section{Funding}

This work was supported by the National Natural Science Foundation of China (No. 81872375, No. 81572655, and No. 8187111248), the Natural Science Foundation of Guangdong Province (No. 2014A050503033, No. 2016A050502011, and No. 2017B020228003), and the National Outstanding Youth Science Fund Project of the National Natural Science Foundation of China (No. 31900461).

\section{Notes}

Role of the funder: The funding sources had no role in the design and conduct of the study; the collection, management, analysis, and interpretation of the data; the preparation, review, and approval of the manuscript; or the decision to submit the manuscript for publication.

Disclosures: The authors have no conflicts of interest to disclose.

Author contributions: X Lv, X Guo and GT Xie were responsible for the study design. MY Qiang, CF Li, YY Sun, Y Sun, LR Ke, T Zhang, YX Li and X Li drafted the manuscript. YY Sun, CM Xie, YJ Zou, WZ Qiu, MY Gao, ZJ Zhan, KY Liu, X Chen, CX Liang, QY Chen and HQ Mai participated in the data interpretation. MY Qiang, CF Li, YY Sun and T Zhang participated in the data collection and analysis. All authors have read and approved the final manuscript.

Acknowledgments: We sincerely thank Yingxue Li (Ping An Healthcare Technology, Beijing, China) and Yuyao Sun (Ping An 
Healthcare Technology, Beijing, China) for building the XGBoost model.

\section{Data Availability}

The authenticity of this article has been validated by uploading the key raw data onto the Research Data Deposit (RDD) public platform (www.researchdata.org.cn), with the approval RDD number RDDA2020001422.

\section{References}

1. Bray F, Ferlay J, Soerjomataram I, Siegel RL, Torre LA, Jemal A. Global cance statistics 2018: GLOBOCAN estimates of incidence and mortality worldwide for 36 cancers in 185 countries. CA Cancer J Clin. 2018;68(6):394-424.

2. Pan J, Ng W, Zong J, et al. Prognostic nomogram for refining the prognostication of the proposed 8th edition of the AJCC/UICC staging system for nasopharyngeal cancer in the era of intensity-modulated radiotherapy. Cancer. 2016;122(21):3307-3315.

3. Sun X, Su S, Chen C, et al. Long-term outcomes of intensity-modulated radiotherapy for 868 patients with nasopharyngeal carcinoma: an analysis of survival and treatment toxicities. Radiother Oncol. 2014;110(3):398-403.

4. DG P, S S. Head and Neck Cancers, Version 2.2020, NCCN Clinical Practice Guidelines in Oncology. J Natl Compr Canc Netw. 2020;18(7):873-898.

5. Tang XR, Li YQ Liang SB, et al. Development and validation of a gene expression-based signature to predict distant metastasis in locoregionally advanced nasopharyngeal carcinoma: a retrospective, multicentre, cohort study. Lancet Oncol. 2018;19(3):382-393.

6. Peng H, Dong D, Fang MJ, et al. Prognostic value of deep learning PET/CTbased radiomics: potential role for future individual induction chemotherapy in advanced nasopharyngeal carcinoma. Clin Cancer Res. 2019;25(14) 4271-4279.

7. Dong D, Zhang F, Zhong LZ, et al. Development and validation of a novel MR imaging predictor of response to induction chemotherapy in locoregionally advanced nasopharyngeal cancer: a randomized controlled trial substudy (NCT01245959). BMC Med. 2019;17(1):190.

8. Chen FP, Wen DW, Li F, et al. The role of post-neoadjuvant chemotherapy tumor volume for prognostication and treatment guidance in loco-regionally advanced nasopharyngeal carcinoma. Cancers (Basel). 2019;11(11):1632.

9. Mao YP, Xie FY, Liu LZ, et al. Re-evaluation of 6th edition of AJCC staging system for nasopharyngeal carcinoma and proposed improvement based on magnetic resonance imaging. Int J Radiat Oncol Biol Phys. 2009;73(5): 1326-1334.

10. Wu S, Zheng J, Li Y, et al. Development and validation of an MRI-based radiomics signature for the preoperative prediction of lymph node metastasis in bladder cancer. EBioMedicine. 2018;34:76-84.

11. Zhang Y, Liu X, Zhang Y, et al. Prognostic value of the primary lesion apparent diffusion coefficient (ADC) in nasopharyngeal carcinoma: a retrospective study of 541 cases. Sci Rep. 2015;5(1):12242.
12. Law BK, King AD, Bhatia KS, et al. Diffusion-weighted imaging of nasopharyngeal carcinoma: can pretreatment DWI predict local failure based on long-term outcome? AJNR Am J Neuroradiol. 2016;37(9):1706-1712.

13. Tang LQ Li CF, Li J, et al. Establishment and validation of prognostic nomograms for endemic nasopharyngeal carcinoma. J Natl Cancer Inst. 2016;108(1): djv291.

14. Huang CL, Sun ZQ Guo R, et al. Plasma Epstein-Barr Virus DNA load after induction chemotherapy predicts outcome in locoregionally advanced nasopharyngeal carcinoma. Int J Radiat Oncol Biol Phys. 2019;104(2):355-361.

15. Wang Y, Liu C, Zhang X, Deng W. Synthetic CT generation based on T2 weighted MRI of Nasopharyngeal Carcinoma (NPC) using a Deep Convolutional Neural Network (DCNN). Front Oncol. 2019;9:1333.

16. Liang S, Tang F, Huang X, et al. Deep-learning-based detection and segmentation of organs at risk in nasopharyngeal carcinoma computed tomographic images for radiotherapy planning. Eur Radiol. 2019;29(4):1961-1967.

17. Chen T, Guestrin C, XGBoost: A scalable tree boosting system. Paper presented at 22nd ACM SIGKDD International Conference on Knowledge Discovery and Data Mining; August 2006; San Francisco, CA

18. Chen T, Li X, Li Y, et al. Prediction and risk stratification of kidney outcomes in IgA nephropathy. Am J Kidney Dis. 2019;74(3):300-309.

19. Zhang Z, Ho KM, Hong Y. Machine learning for the prediction of volume responsiveness in patients with oliguric acute kidney injury in critical care. Crit Care. 2019;23(1):112.

20. MB A. American Joint Committee on Cancer. In: AJCC Cancer Staging Manual. 8th ed. New York: Springer; 2017.

21. Harrell FE, Jr, Califf RM, Pryor DB, Lee KL, Rosati RA. Evaluating the yield of medical tests. JAMA. 1982;247(18):2543-2546.

22. Heagerty PJ, Lumley T, Pepe MS. Time-dependent ROC curves for censored survival data and a diagnostic marker. Biometrics. 2000;56(2):337-344.

23. Schröder MS, Culhane AC, Quackenbush J, Haibe-Kains B. survcomp: an R/ Bioconductor package for performance assessment and comparison of survival models. Bioinformatics. 2011;27(22):3206-3208.

24. Haibe-Kains B, Desmedt C, Sotiriou C, Bontempi G. A comparative study of survival models for breast cancer prognostication based on microarray data: does a single gene beat them all? Bioinformatics. 2008;24(19):2200-2208.

25. Chen YP, Chan ATC, Le QT, Blanchard P, Sun Y, Ma J. Nasopharyngeal carcinoma. Lancet. 2019;394(10192):64-80.

26. Zhang B, Tian J, Dong D, et al. Radiomics features of multiparametric MRI as novel prognostic factors in advanced nasopharyngeal carcinoma. Clin Cancer Res. 2017;23(15):4259-4269.

27. Ming X, Oei RW, Zhai R, et al. MRI-based radiomics signature is a quantitative prognostic biomarker for nasopharyngeal carcinoma. Sci Rep. 2019;9(1):10412.

28. Jiang Y, Qu S, Pan X, Huang S, Zhu X. Prognostic nomogram for locoregionally advanced nasopharyngeal carcinoma. Sci Rep. 2020;10(1):861.

29. Lan X-W, Xiao Y, Zou X-B, Zhang X-M, OuYang P-Y, Xie F-Y. Outcomes of adding induction chemotherapy to concurrent chemoradiotherapy for stage T3N0-1 nasopharyngeal carcinoma: a propensity-matched study. Onco Targets There. 2017;10:3853-3860.

30. LR W, HL Y, NJ, et al. Prognostic value of chemotherapy in addition to concurrent chemoradiotherapy in T3-4N0-1 nasopharyngeal carcinoma: A propensity score matching study. Oncotarget. 2017;8(44):76807-76815.

31. Liang H, Xiang YQ, Lv X, et al. Survival impact of waiting time for radical radiotherapy in nasopharyngeal carcinoma: a large institution-based cohort study from an endemic area. Eur J Cancer. 2017;73:48-60. 